\title{
EDITORIAL \\ Technology around the world: eight countries, shared problems
}

\author{
Michael Seadle and Elke Greifeneder \\ Berlin School of Library and Information Science, Humboldt University, \\ Berlin, Germany
}

\begin{abstract}
Purpose - This editorial aims to discuss how technology has transformed the cultural map for libraries so that experiences in Taiwan, Iran, India, Greece, or New Zealand are relevant anywhere in the developed world.

Design/methodology/approach - The methodology draws heavily on cultural anthropology in looking at the cultural context and its changes over time.

Findings - Four sets of shared problems emerge in these articles: technology applications to solve specific library problems, user studies, cataloging issues, and electronic publishing. These are topics that librarians discuss around the world.

Originality/value - It is a commonplace today that technology has tended to equalize widely separated parts of the world. This is obviously true in terms of consumer goods, but appears to be less well accepted in terms of scholarly endeavor. The international and intellectual breadth of this issue can be seen as something to celebrate.
\end{abstract}

Keywords Libraries, Communication technologies

Paper type General review

\section{Introduction}

The technology-related library literature in the English speaking world tends to concentrate on problems and examples that come from countries where English is the dominant language. This is not surprising, but it leads to a tendency to neglect the rest of the world - or to assume that the rest of the world is merely catching up and has nothing of interest for English speaking lands.

If we take an anthropological perspective on the use of technology tools around the world, it is clear that the tendency to focus inward has had a rational basis, not merely for English-speaking countries but for most technology cultures. During the dawn of computer culture in the 1950s, computer systems and computing languages led a short and isolated existence. Machines from different manufacturers had incompatible chip-sets, and high-level languages like Fortran, which could run on multiple computing platforms with the help of an appropriate compiler, had only started to be used.

By the 1970s and 1980s interoperability grew to the point that some machines from different manufacturers could share both operating systems and application programs by physically transporting a storage medium like punch cards or magnetic tape. The Internet existed at this time too, though only for a very small research community. Sharing programs and problems occurred on a limited basis within groups that defined themselves by location and manufacturer. Those working on IBM S360/379 style machines had little practical interaction with those using DEC, Burroughs, Sperry-Rand,

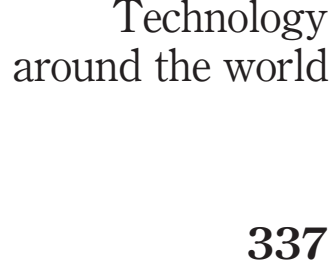

Received 11 March 2008 Revised 13 March 2008 Accepted 16 March 2008

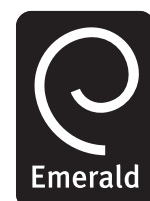

Library Hi Tech Vol. 26 No. 3, 2008 pp. $337-341$ (C) Emerald Group Publishing Limited 0737-8831 
LHT

26,3

338
Siemens or other machines. Some computers in the Soviet zone emulated western models, but others had their own internal structure. Communication across the political barrier of the "iron curtain" was essentially nonexistent. Computer use in Africa and Asia suffered from a lack of steady electric power and from a shortage of trained programming staff. In other words, the situation before the 1990s fit a mental model where what went on in computing technology outside the English speaking world hardly mattered to anyone inside it, especially since the manufacturer-based divisions within were so great.

The growth of the Web and the increasing reliance on the Internet for interactive applications and information discovery has changed the technological culture map. That does not mean that everything has become identical. Languages that use different scripts, for example, create different problems in display and in searching. Unicode can solve many of the display issues, but it does not resolve the grammatical assumptions build into search engines designed for western language texts. Linux, MS-Windows, Macintosh OS-X all provide the basic computing infrastructure for contemporary systems that link to the Internet and share resources like Google, del.icio.us, Blogspot, or YouTube. Nonetheless many problems are similar. In this issue we have collected a number of examples.

\section{Today's technology culture map}

A visual representation of the map of contemporary library technology would likely have the Anglo-American-Canadian world at its center, since the resources for development remain significantly greater there than elsewhere. The Anglo-American-Canadian universities continue to attract people from all parts of the world, which helps to drive this development process. They also simply have more money, largely because of the longstanding private and endowment funding that North American institutions have built up, especially over the past several decades.

Next to this core in the technology map sit western European countries like The Netherlands, Germany, France, Scandinavia, and Asian countries like Japan, Taiwan, and Korea. A host of smaller countries with English as an official language appear in this zone as well, including Singapore, Ireland, Australia, New Zealand and South Africa. Giants like India, China, Brazil occupy a third ring where resources are less even, but at their best they are equal to those in the inner core. Eastern and southern European countries probably have a place here, as do places like Iran, Pakistan, Botswana, or Nigeria whose infrastructure and intellectual capital make them competitors.

What is particularly striking about this culture map is the degree of technological as well as social interconnection. A librarian in India with an Internet connection can make the same use of Google Scholar as a librarian at a public library in California. There will certainly be differences in what for-cost resources can be licensed, but those are add-ons, not core capabilities. The articles in this issue present a broad sweep of topics. They were not chosen for thematic consistency, but rather for their breadth. In the section below we will explain why each presents a problem of interest to Library Hi Tech readers.

From an anthropological viewpoint it is particularly important to note the differences as well. Technology is only one factor in how humans interact with Internet-based resources. Culture matters too and the cultural diversity in countries like the US, UK, 
or Germany to an increasing extent mirrors the variety in technologically connected countries within those circles in the map above.

\section{Shared problems}

Four sets of shared problems emerge in these articles: technology applications to solve specific library problems, user studies, cataloging issues, and electronic publishing. These are topics that librarians discuss around the world. All four had substantial coverage at the German Library Association conference in Mannheim, Germany, in June 2008, and the program for the American Library Association annual meeting in Anaheim, California, gives them equal attention. For some topics local conditions play a significant role. This is true, for example, for Anna Koulikourdi's article on "Assistive technologies in Greek libraries" as well as Ma Lei Hsieh's US-based article on "A university library laptop lending service: an analysis using two student surveys" but location does not matter for Seda Ozmutlu's article on "Analyzing the results of automatic new topic identification”.

\section{Technology applications}

Library Hi Tech has a long tradition of publishing articles on assistive technologies but has not had any articles specifically on the problems in Greece. The author writes:

[...] there is no intention to compare the suppliers (production and delivery systems) of the AT market and the libraries of Greece and those abroad [. . . Global suppliers in the AT field and international libraries have been ahead of us in these fields for years [...]

This key problem is one that many countries share. The market share is smaller for countries that do not use the standard Latin-based ASCII alphabet, but the use of local languages matters, especially for end-user acceptance.

RFID represents another topic on which we have published articles regularly. Shien-Chiang Yu's article from Taiwan on "Implementation of an innovative RFID application in libraries" offers several innovative ideas, including the use of Ultra High Frequency $(900 \mathrm{MHz})$ to enable signals over a much longer distance, and to use the results to analyze floor planning and seat access. It is clear that the author is not writing from a poor country. He warns readers that if the budget is tight, "it is not cost-efficient for the library to use RFID tags only to replace the functions of a barcode".

\section{User studies}

Libraries around the world recognize today that understanding users is key to their success, perhaps even to their continuation. Yazdan Mansourian's methodology in his article from Iran on "Contextual elements and conceptual components of information visibility on the web" is broadly applicable. He explains that most information literacy articles focus on the role of resources and contents in satisfying a users' information needs and give less attention to key affective and cognitive aspects of the process.

In her article "Building participative library services: the impact of social software use in public libraries" Luise Rutherford used a qualitative methodology that took the form of open-ended interview questions using an e-mail format. She comes from New Zealand, which, though small and isolated, is of course an English speaking country. Using email for her survey allowed a much broader participant group than any local focus group could achieve. A significant part of the value of this paper is that 
LHT

26,3

340 it provides a resource for public librarians who want to understand the potential impact of implementing social software in their own library; and its credibility is enhanced, despite relatively small numbers, because the breadth of the participants allows readers to learn from the experiences of others.

An inverse example is, in some sense, Ma Lei Hsieh, whose article on "A university library laptop lending service: an analysis using two student surveys" examines a very specific local setting in New Jersey and a very focused set of users at Monmouth University. This study used a survey methodology and looked specifically at how libraries could implement laptop lending services. She focuses on the student perspective.

\section{Cataloging issues}

Cataloging is a particularly international activity today. The issue is less what metadata format to use than how to harvest the information for the metadata efficiently and intelligently. Mehri-e-Sedighi's article from Iran on the "Use of geographical information system (GIS) in cataloging of documents: A case study of earthquake documents collections" offers a new tool to illustrate, analyze and review data about the geographical dispersion of earthquakes. It also helps to identify research gaps in various geographic regions.

Topic changes within a user search session is a key issue in content analysis of search engine user queries. New topic identification is an issue in analyzing search engine transaction logs, and particularly estimating topic shifts. The study by Seda Ozmutlu and Gencer C. Cosar from Turkey in "Analyzing the results of automatic new topic identification" looks at subject shifts within a user search session. Tracking these changes leverages computing power to help librarians understand how our descriptive choices in catalog records affect the efficiency of search results.

\section{Electronic publishing}

University libraries in the Anglo-American world have recognized that the future belongs to electronic copies of journals and that the terms for acquiring complete sets of journals from a publisher (often called the "big deal") represents a financial advantage over individual journal subscriptions. Margam Madhusudhan addresses the use of these electronic journal packages in his article on the "Use of UGC-Infonet e-journals by research scholars and students of the University of Delhi, Delhi: a study". His study looks specifically at the information needs of students in library and information science at the University of Delhi, including fundamentals like whether the students know about the existence of the resources, whether they have access to them, and whether they make use of all available search strategies. One particularly interesting result is that $100 \%$ "of the respondents admit that e-journals are good substitutes for conventional resources" if reasonable access via sufficient terminals with high speed access is available.

While electronic journals have an established place in research libraries, electronic books are less firmly settled. Many public domain books have become available through cooperative efforts like Project Gutenberg (www.gutenberg.org/) or the Universal Digital Library/Million Books project (www.ulib.org/), but precisely how we distinguish an e-book from other digital aggregations is not entirely clear. Magda Vassiliou and Jennifer Rowley from England use a content analysis method to examine 37 e-book 
definitions to find the common elements. They offer a two part conclusion, one based on existing social concepts of the traditional content of a print publication, and the other on technology that includes "features such search and cross reference functions, hypertext links, bookmarks, annotations, highlights, multimedia objects and interactive tools." The authors emphasize that the definition, especially the technology aspect, is dynamic and can change over time.

Paul Conway's article on "Modeling the digital content landscape in universities" provides a synthesis ofhow librarians can conceptualize the environment in which most Technology
around the world serious contemporary research libraries function. He proposes a "multi-dimensional framework that addresses three outstanding issues with digital asset management in universities." It balances mission, selection process, and management priorities for various classes of digital content:

More specifically the Conway Content Landscape Model articulates four interacting variables that together describe the core asset management challenges that universities face with digital content: property rights, structure, source and possession.

While the model has been applied mainly at Duke University in the US, its structure can be used at any university research library in the world.

\section{Conclusion}

It is a commonality today that technology has tended to equalize widely separated parts of the world. This is obviously true in terms of consumer goods, but appears to be less well accepted in terms of scholarly endeavor. Journals tend mainly to draw authors from the countries where they started, where they are published, and where their editorial offices are located. It is relatively rare that a journal's editorial offices migrate from country to country and continent to continent, as has Library Hi Tech. It is also relatively rare that an established and successful journal looks to take risks on new and unknown authors or topics that might step beyond the workday world of the common reader.

As editors we see the international and intellectual breadth of this issue as something to celebrate. When Ed Wall founded the journal in Michigan 26 years ago he saw it as a vehicle for a new way of looking at the technology that had started to transform libraries. Today in Berlin we are no less open to transformative topics and new sources. Our readership is world-wide and our interests are as well.

\section{Corresponding author}

Michael Seadle can be contacted at: seadle@ibi.hu-berlin.de

\footnotetext{
To purchase reprints of this article please e-mail: reprints@emeraldinsight.com
} Or visit our web site for further details: www.emeraldinsight.com/reprints 